\title{
Nuevos escenarios para la comunicación comunitaria. Oportunidades y amenazas a medios de comunicación y organizaciones de la sociedad civil a partir de la aplicación del nuevo marco regulatorio ecuatoriano
}

Natalia García ${ }^{1}$
Caroline Ávila

Recibido: 2015-03-26

Enviado a pares: 2015-03-26
Aprobado por pares: 2015-05-12

Aceptado: 2015-05-27

DOI: 10.5294/pacla.2016.19.1.11

Para citar este artículo / to reference this article / para citar este artigo

García, N. \& Ávila, C. (Marzo de 2016). Nuevos escenarios para la comunicación comunitaria. Oportunidades y amenazas a medios de comunicación y organizaciones de la sociedad civil a partir de la aplicación del nuevo marco regulatorio ecuatoriano. Palabra Clave, 19(1), 271-303. DOI: 10.5294/pacla.2016.19.1.11

\section{Resumen}

Esta investigación analiza el escenario actual de los medios comunitarios y alternativos con relación al marco regulatorio ecuatoriano recientemente aplicado a medios de comunicación y organizaciones de la sociedad civil. Para empezar, se establece un marco teórico basado en la historia de medios comunitarios y alternativos y organizaciones de la sociedad civil en Latinoamérica y Ecuador. Con esta información se describe y analiza el nuevo marco regulatorio (Ley de Comunicación y Decreto Ejecutivo n. $\left.^{\circ} 16\right)$ a través de las opiniones de expertos y de los alcances que han

Universidad de Azuay, Ecuador. nataliagarciafreire@gmail.com

Universidad de Azuay, Ecuador. cavila@uazuay.edu.ec 
tenido estos instrumentos legales en tres casos específicos: Radio La Voz de Ingapirca, Radio Sonoonda, y la confederación de pueblos de nacionalidad kicwhua de Ecuador, Ecuarunari. Con la descripción de este escenario se establecen las bases para poder analizar la aplicación de este marco regulatorio que constituye, en cierto modo, un nuevo paradigma en regulación de la comunicación.

\section{Palabras clave}

Medios comunitarios, legislación de las comunicaciones, sociedad civil, democratización de la comunicación, economía política de la comunicación (Fuente: Tesauro de la Unesco). 


\section{New Scenarios for Community Communication. Opportunities and Threats to the Media and Civil Society Organizations from the Application of the New Ecuadorian Regulatory Framework}

\section{Abstract}

This research analyzes the current situation of community and alternative media in relation to Ecuadorian regulatory framework recently applied to media and civil society. To start a theoretical framework based on the history of community and alternative media and civil society organizations in Latin America and Ecuador is established. This information describes and analyzes the new regulatory framework (Communications Law and Executive Decree No. 16) through expert opinions and achievements that these legal instruments have had in three specific cases: Radio La Voz de Ingapirca, Radio Sonoonda, and the Confederation of towns of Kicwhua nationality Ecuador, Ecuarunari. With the description of this scenario the bases to review the implementation of this regulatory framework are, in a way, a new paradigm in establishing communication regulations.

\section{Keywords}

Community media, communications law, civil society, democratization of communication, political economy of communication (Source: Unesco Thesaurus). 


\section{Novos cenários para a comunicação comunitária. Oportunidades e ameaças a meios de comunicação e organizações da sociedade civil a partir da aplicação do novo marco regulatório equatoriano}

\section{Resumo}

Esta pesquisa analisa o cenário atual dos meios comunitários e alternativos a respeito do marco regulatório equatoriano recentemente aplicado a meios de comunicação e organizações da sociedade civil. Para começar, estabelece-se um referencial teórico baseado na história de meios comunitários e alternativos, bem como de organizações da sociedade civil na América Latina e no Equador. Com essa informação, descreve-se e analisa-se o novo marco regulatório (Lei de Comunicação e Decreto Executivo 16) por meio das opiniões de especialistas e dos progressos que esses instrumentos legais vêm tendo em três casos específicos: Radio La Voz de Ingapirca, Radio Sonoonda e a Confederação De Povos de Nacionalidade Kicwhua do Equador (Ecuarunari). Com a descrição desse cenário, estabelecem-se as bases para poder analisar a aplicação desse marco regulatório que constitui, em certa medida, um novo paradigma em regulação da comunicação.

\section{Palavras-chave}

Meios comunitários, legislação das comunicações, sociedade civil, democratização da comunicação, economia política da comunicação (Fonte: Tesauro da Unesco). 


\section{Introducción}

En el actual debate sobre la comunicación en Latinoamérica en general y en Ecuador en particular, desde la Ley de Comunicación hasta las actuales enmiendas que buscan garantizar la comunicación como un servicio público, cabe resaltar la consideración de los medios comunitarios como un avance en materia de legislación en Ecuador. Sin embargo, aunque la nueva ley reconozca la figura jurídica de un medio comunitario, es preciso saber cómo otros recientes marcos regulatorios influyen en este escenario.

La Ley de Comunicación en Ecuador aprobada en 2013 plantea que los medios comunitarios representen el $34 \%$ de las frecuencias totales en radio y televisión. Para este fin, la ley establece en varios artículos que existirá el apoyo total por parte del Estado (Ley Orgánica de Comunicación, 2013). Por otro lado, desde 2009 ha aumentado el rigor en el control a las organizaciones de la sociedad civil (OSC), que culminó con la aplicación del Decreto Ejecutivo n. ${ }^{\circ} 16$ en 2013.

La historia de medios comunitarios nos permite ver que estos siempre han tenido relación con organizaciones de la sociedad civil, tanto en su creación como en su desarrollo. Estos medios son los portavoces de la sociedad civil, pues muchos han nacido de organizaciones sociales y comunidades (Gumucio, 2005).

Los principios para un marco regulatorio de medios comunitarios establecidos por la Asociación Mundial de Radios Comunitarias (Amarc) dejan claro que se debe reconocer la figura jurídica de medios comunitarios y garantizar su existencia en un estado; sin embargo, también aclaran que se debe garantizar una efectiva participación de la sociedad civil en los procesos de toma de decisiones.

Por tanto, esta investigación busca analizar el escenario actual de los medios comunitarios y alternativos con relación a los marcos regulatorios de medios y organizaciones de la sociedad civil. Para hacerlo, se establecerá el marco contextual bajo el cual los medios comunitarios nacen como parte de la sociedad civil, y por lo tanto mantienen estrecha relación con 
otras OSC. Se analizarán los marcos regulatorios (Ley de Comunicación y Decreto Ejecutivo n. $\left.{ }^{\circ} 16\right)$ a través de las opiniones de expertos y de los alcances que han tenido hasta ahora. Por último, se revisará su aplicación en diferentes ejemplos relevantes para el caso.

Con esta investigación se pretende contribuir al análisis de los desafíos para la comunicación comunitaria como parte de este nuevo paradigma que propone Ecuador en materia de regulación y política pública en comunicación.

\section{Marco contextual}

\section{Medios comunitarios y sociedad civil}

Hablar de medios comunitarios supone una gran responsabilidad, pues se habla de un concepto que engloba —más allá de medios o herramientas de comunicación - expresiones sociales, ideológicas, políticas y culturales, una voz alternativa inherente a la sociedad (Gumucio, 2010). Entender los medios comunitarios es — de la misma manera - una tarea difícil: cada experiencia es diferente, cada una es una respuesta a una lucha social, a una necesidad de la comunidad, a un contexto histórico. Legislar los medios comunitarios es entonces una tarea compleja que implica responsabilidad y un entendimiento profundo de ellos y de sus características.

Para empezar a hablar de estos medios hay que acercarse a la historia y reconocer que no se puede hablar de ellos sin hablar de sociedad civil, o de ciudadanía. Fernanda Corrales e Hilda Hernández (2009), en su estudio sobre comunicación alternativa en nuestros días, mencionan que los medios alternativos o comunitarios han sido inherentes al desarrollo de la sociedad y han existido desde siempre. Sin embargo, aclaran que no es sino hasta la segunda mitad del siglo XX que se da un auge y empieza a verse en todo el mundo este nuevo tipo de medios de comunicación: los medios alternativos o comunitarios. ¿Qué sucede alrededor de esta época?

En América Latina, las décadas de los sesenta, setenta y ochenta cuentan una historia de dictaduras represivas en Chile, Argentina, Uruguay y 
Brasil; democracias precarias en Venezuela y Colombia, y democracias de dominio militar en Perú y Ecuador (Anderson, 1987). En este contexto se encuentra el cierre de canales institucionales de expresión, y represión política y social. Bajo estos parámetros, el término "sociedad civil" empieza a invocarse con mayor fuerza cada vez (Jelin, 1994). De la mano del término surgen nuevas formas y actores sociales que reaccionan ante la represión del estado, la falta de participación ciudadana y el desmantelamiento de organizaciones sociales. Estas nuevas formas sociales son variadas y van desde grupos paramilitares, grupos juveniles, ONG, fundaciones y comunidades indígenas hasta radios comunitarias (Dagnino, 2011).

La época siguiente está marcada por un retorno a la democracia. Este proceso va de la mano de una corriente antiestatista y una ideología neoliberal. Sin embargo, al contrario de lo que se pensaría, la transición a la democracia no logra sus cometidos. Los países de la región latinoamericana tienen dificultades para conseguir la vigencia de sus derechos económicos y sociales. Si bien la realidad de las dictaduras ha quedado atrás, persisten las violaciones de derechos civiles elementales y las minorías sufren discriminaciones institucionales, en tanto el neoliberalismo no cumple las promesas de una "ideal democracia". Las formas sociales, antes mencionadas, surgen como una respuesta a este contexto y buscan construir una democracia desde abajo y pensar soluciones de base, no desde el estado o la empresa privada sino desde la ciudadanía, desde la sociedad civil (Jelin, 1994).

La realidad mundial en estas épocas está marcada también por otra característica: un retorno a las miradas hacia los derechos humanos. En este contexto se empieza a hablar en Latinoamérica de derechos de los indígenas, derechos de la mujer, derechos del niño. Hablar de esto es reconocer que ha habido una discriminación hacia las minorías y, además, tener un compromiso de cambio (Jelin, 1994). Adicionalmente, desde los años ochenta viene ya cambiando la forma de lucha social, que ya no se hace desde sindicatos sino a partir de luchas sectorizadas representadas por asociaciones de mujeres, jóvenes e indígenas (Celis y Sánchez, 2011). Hasta los años setenta, la democracia gira en torno al estado y los partidos políticos, pero las nuevas formas sociales cobran importancia en la construcción de 
esta democracia. La mayoría aparecieron alrededor de los años sesenta y setenta, los años de represión, razón por la cual fueron esenciales en los procesos de redemocratización.

Este es el contexto histórico bajo el cual los medios comunitarios nacen como una rama de la sociedad civil, como una herramienta para dar voz a grupos que no habían sido visibilizados.

El término "medio comunitario" puede ser reemplazado por un sinfín de términos que han servido para nombrar a las distintas experiencias que se han dado alrededor del mundo. Se puede hablar así de medios alternativos, radicales, ciudadanos, marginales, participativos, de contrainformación, paralelos, comunitarios, underground, populares, libres, disidentes, de resistencia, piratas, clandestinos, autónomos, jóvenes y micromedios (Rodríguez, 2009), además de medios independientes, tercer sector audiovisual y medios de la sociedad civil (Milan, 2006).

Cada definición responde a características del medio y reflejan lo único de cada experiencia; esto demuestra que, a diferencia de los medios masivos, los medios comunitarios son respuestas puntuales a necesidades sociales. Es curioso además que entre los términos utilizados para designarlos estén piratas o clandestinos, reflejo de una historia que los hizo parecer invisibles durante mucho tiempo.

Hasta hace pocos años, estos medios no tenían reconocimiento legal en Latinoamérica. Esto responde a múltiples factores. Por un lado, las legislaciones de nuestra región se remontaban a gobiernos autoritarios y dictatoriales que únicamente reconocían a medios privados y públicos (Ávila, 2013). Por otro lado, como menciona Chiara Sáez (2008), existió siempre una invisibilización desde la teoría de la comunicación, desde los sistemas de medios y desde la esfera pública. A pesar de estas limitantes, las experiencias de medios comunitarios no solo surgen sino que se multiplican con el paso de los años. En muchos países de la región se crean medios comunitarios a cargo de la iglesia, ONG y universidades o se constituyen jurídicamente como medios privados para conseguir acceso a frecuencias (Gumucio, 2005). 
No es sino hasta inicios del siglo XXI que se empieza a reconocer legalmente la existencia de estos medios en algunos países como Argentina, Ecuador, Uruguay y Bolivia. Además, se los reconoce con fuertes restricciones en otros países como Brasil y Chile, mientras que en países como Guatemala y Paraguay aún se les prohíbe el funcionamiento.

En Latinoamérica esta ola de democratización de la comunicación se da desde principios del año 2000. La sociedad civil forma importantes alianzas que formulan propuestas para exigir cambios en las legislaciones de cada país. Se forma la Coalición por una Radiodifusión Democrática en Argentina en 2004, el foro de Comunicación y Participación ciudadana de Uruguay, la Plataforma por los Derechos de Información y Comunicación en Bolivia en 2007, el Foro Ecuatoriano de la Comunicación en 2007, el Colectivo Ciudadano por los Derechos de la Comunicación en 2009 y finalmente los autoconvocados de la comunicación en 2010. La redistribución tripartita es el resultado de una lucha desde la sociedad civil y los medios comunitarios (Segura, 2014).

\section{Nuevas legislaciones a medios de comunicación y a la sociedad civil: el caso de Ecuador}

Latinoamérica como región presenta un mayor desarrollo del sector de la comunicación comunitaria en comparación con Europa o Norteamérica. Experiencias en países como Brasil, Argentina o Colombia son un claro ejemplo del desarrollo de este sector (Sáez, 2008; Villamayor, 2014; Araujo, 2007; Gueerts, Van Oeyen y Villamayor, 2004). El de Ecuador es un caso interesante porque, como se ha establecido en la sección anterior, estas características llevaron a que en el 2008 se convirtiera en el primer país de América Latina en tener una constitución que reconozca el derecho a la comunicación y establezca una redistribución equitativa entre los sectores privado, público y comunitario. De hecho, a criterio de Segura (2012), es uno de las países en Latinoamérica con el mayor grupo de organizaciones de la sociedad civil dedicadas a la comunicación. Además cuenta con movimientos indígenas que han tenido una fuerte repercusión en su desarrollo político y social. 
El primer esfuerzo por regular la comunicación en Ecuador se remonta a 1976, con la ley creada en el gobierno dictatorial del general Guillermo Rodríguez Lara, en la que se trataba a los medios comunitarios bajo la denominación de medios comunales, se limitaba su cobertura y se prohibía en ellos la publicidad pagada. Además, aquella ley no establecía ningún reglamento para la concesión de frecuencias.

Varias organizaciones de la sociedad civil buscaron reformar esta ley en diversas oportunidades. Uno de esos esfuerzos fue liderado por la Coordinadora de Radios Populares y Alternativas del Ecuador, Corape, que en 1996 buscó una reforma por considerar que discriminaba y minimizaba a los medios comunitarios. En el año 2000, con el apoyo de la Fundación Friedrich Ebert Stifung, la Confederación de Nacionalidades Indígenas del Ecuador (Conaie), la Confederación de Pueblos de la Nacionalidad Kichwa del Ecuador (Ecuarunari) y la diputada Nina Pacari (Pachakutik), Corape presentó varias reformas a esta ley. En 2002, después de varios debates, el Congreso Nacional emitió un informe cuyo resultado fue, al menos, el reconocimiento de la existencia de medios comunitarios en nuestro país y el derecho a acceder a publicidad pagada. Sin embargo, aún se incluyó a estos medios dentro del sector de medios públicos (ALER, 2011).

En 2008, con la nueva constitución, como se mencionó anteriormente, se logró el reconocimiento del sector comunitario, pero no fue sino hasta 2013, con la aprobación de la Ley de Comunicación, que se sentaron las bases para un nuevo escenario de los medios comunitarios.

El establecimiento de ese nuevo escenario no estuvo exento de presiones desde diferentes sectores. Como lo explica el informe de la Asociación Latinoamericana de Educación Radiofónica (ALER), desde 2009 organizaciones de la sociedad civil como Corape, la misma ALER, el Centro Internacional de Estudios Superiores de Comunicación para América Latina (Ciespal), Radialistas Apasionados, Ecuaranari, la Corporación de Emisoras de las Nacionalidades de Ecuador (Cenae), la Gestión Social para el Desarrollo (GSD), la Agencia Latinoamericana de Información (ALAI) y la Asociación para el Progreso de las Comunicaciones han celebrado varias 
reuniones y asambleas, una de ellas con el secretario de Comunicación del Estado, Fernando Alvarado, en la que se firmó un acuerdo para que se incluya la distribución equitativa y progresiva del espectro en tres tercios para los sectores de la comunicación, entre otros puntos importantes (ALER, 2011).

Asimismo, en esta asamblea se conformó la comisión ocasional de comunicación para tratar el tema de la Ley de Comunicación. En uno de los debates se propuso un $25 \%$ del espectro radioeléctrico para medios comunitarios, sin embargo las organizaciones no dieron marcha atrás y exigieron siempre el $33 \%$. Desde la sociedad civil se sugirió la creación de un consejo de regulación conformado por el ejecutivo, un integrante postulado por la Asamblea Nacional, un integrante postulado por el Consejo de Igualdad, un integrante postulado por las facultades o escuelas de comunicación social y tres representantes de la ciudadanía (ALER, 2011).

Entre 2010 y 2013 continuaron los debates y discusiones en torno a la Ley de Comunicación en Ecuador. La ley, aprobada en junio de 2013, representó una gran victoria para la comunicación comunitaria, ya que establecía que el 34 \% del espectro radioeléctrico sería destinado a los medios comunitarios.

Como se menciona en varios estudios, esta victoria fue el resultado de años de lucha por parte de organizaciones sociales, académicos, medios comunitarios y alternativos que han tenido lugar, en el caso ecuatoriano, desde los años setenta. Sin embargo, cuestiones como el consejo de regulación y otros aspectos tomaron un rumbo distinto en la ley.

Junto con la creación de la Ley de Comunicación, otro proceso se daba de manera paralela pero mucho más silenciosa: en 2013, el gobierno ecuatoriano emitió el Decreto Ejecutivo n. ${ }^{\circ}$ 16, que regula a las organizaciones de la sociedad civil. Este hecho causó preocupación en representantes nacionales de diferentes organizaciones no gubernamentales y también en organismos internacionales debido a las implicaciones que podría generar. Hasta la fecha, este decreto tiene cuatro demandas de inconstitucionalidad 
y ha recibido fuertes críticas por parte de organizaciones internacionales como la CIDH y Human Rights Watch.

De la estrecha vinculación entre las organizaciones de la sociedad civil, ahora reguladas por el Decreto Ejecutivo n. ${ }^{\circ} 16$, y los medios comunitarios, regulados por la Ley Orgánica de Comunicación (LOC), surgen las interrogantes que intenta responder esta investigación:

P1: ¿Cuál es el escenario para la comunicación comunitaria en Ecuador con estos nuevos marcos regulatorios?

La descripción del escenario implica también el análisis desde la perspectiva de los expertos y de los actores, razón por la cual se establece la segunda interrogante:

P2: ¿Cómo influye la Ley de Comunicación y el Decreto Ejecutivo n. ${ }^{\circ} 16$ en el escenario de los medios comunitarios en Ecuador?

Esta segunda interrogante nos permite plantear la siguiente hipótesis de trabajo:

H1: Existe una contradicción entre la nueva Ley de Comunicación y el Decreto Ejecutivo n. ${ }^{\circ} 16$ que influye negativamente en el escenario de los medios comunitarios en nuestro país.

\section{Metodología}

Esta investigación toma como referencia teórica la sustentabilidad de los medios comunitarios planteada por Gumucio (2009), junto con los postulados sobre comunicación comunitaria y esfera pública alternativa de Sáez (2008). Desde un enfoque cualitativo, utiliza el estudio de caso como metodología de investigación, a través de la revisión bibliográfica y de fuentes secundarias en combinación con entrevistas semiestructuradas como técnicas de recolección (Yin, 2009; King, Keohane y Verba, 1994). Trabajos similares han acogido este enfoque metodológico para el análisis del estudio de caso (Araujo, 2007; Gueerts, Van Oeyen y Villamayor, 2004). 
Esta investigación tiene un alcance descriptivo-correlacional. Su fin es mostrar el escenario actual legal que rige a medios y organizaciones de la sociedad civily cómo este escenario influye en los medios comunitarios.

La investigación bibliográfica permitió definir un marco de análisis sobre los conceptos de comunicación comunitaria, sustentabilidad, sociedad civil, ONG y democratización de la comunicación. Posteriormente se hizo una revisión histórica de la comunicación comunitaria en Ecuador y los aspectos convergentes con las organizaciones de la sociedad civil. Para el análisis de archivo se consideraron estudios realizados por organizaciones como Ciespal, Amarc, Radialistas Apasionados, ALER, Corape y Unesco, entre otras. La revisión de literatura y fuentes secundarias se complementa con aproximadamente diez entrevistas semiestructuradas realizadas a abogados involucrados en la elaboración del marco regulatorio vigente en Ecuador y a expertos comunicadores (entre ellos catedráticos universitarios, investigadores de la comunicación y radiodifusores). Fueron también entrevistados representantes de organismos no gubernamentales (Fundamedios, Participación Ciudadana), de organizaciones comunitarias (Corape, Ecuarunari, FOA) y del sector gubernamental (Consejo de Regulación y Desarrollo de la Información - Cordicom-y Superintendencia de Comunicación). Los entrevistados fueron escogidos por su perfil y grado de involucramiento con el objeto de estudio.

Posteriormente se recogieron ejemplos puntuales en los que se evidencia la influencia del marco regulatorio en la situación actual de la comunicación comunitaria en Ecuador. En este caso se aplica lo planteado por Gueerts et al. (2004) al escoger el caso de estudio de acuerdo con la riqueza de la experiencia de la que se pueda obtener la mayor cantidad de información (Flyvbjerg, 2006; Navarro, 2011). Las dos experiencias escogidas son emisoras que están involucradas en este estado convergente del marco regulatorio, es decir, son organizaciones no gubernamentales que hacen comunicación comunitaria y que deben regularizar su situación de acuerdo con el nuevo marco legal. Existe una tercera experiencia asociada con la organización comunitaria no gubernamental, que busca conseguir una frecuencia comunitaria. 
La información conseguida, que combina el análisis del contexto y los tres casos de estudio, permitió construir el escenario en torno a medios comunitarios, ya que se incorporaron voces de un amplio espectro de los sectores representativos del contexto (academia, radiodifusión, organizaciones de la sociedad civil, asociaciones comunitarias, sector gubernamental, abogados e investigadores). El estudio de caso, como estrategia metodológica, permite establecer análisis y conclusiones de una forma flexible y sistémica, ya que se observan los fenómenos en su contexto y como un todo. El proceso iterativo entre la literatura, las fuentes secundarias y las entrevistas y observaciones permiten darle confiabilidad y validez a la información; sin embargo, es necesario reconocer que sus resultados pertenecen a un contexto y circunstancias específicas que, en este caso, ilustran una realidad observada como consecuencia de un nuevo marco regulatorio (Sáez, 2008; Yin, 2009; King, Keohane y Verba, 1994; Flyvbjerg, 2006).

\section{Análisis y discusión}

\section{La Ley Orgánica de Comunicación de Ecuador}

La ley aprobada en junio de 2013 es un cuerpo de 119 artículos distribuidos en seis títulos, 26 disposiciones transitorias, seis reformatorias y dos derogatorias. Su objetivo es desarrollar, proteger y regular el ejercicio de los derechos de la comunicación establecidos en la constitución ecuatoriana. Este instrumento establece elementos conceptuales sobre comunicación, principios y derechos, entre ellos los derechos de los comunicadores, niños y adolescentes, el acceso a la información, la interculturalidad, etc. Establece también un sistema de comunicación con connotaciones ambiguas y criticadas por su fuerte énfasis en la regulación a los miembros del sistema. Define a los medios de comunicación desde la triada de medios públicos, privados y comunitarios; en ese marco distribuye el espectro radioeléctrico en $33 \%$, $33 \%$ y $34 \%$, respectivamente. La ley finaliza con secciones que regulan la publicidad, la producción audiovisual y los espectáculos públicos (Ávila, 2013).

En la declaración conjunta sobre diversidad de radiodifusión de 2007 realizada por los relatores de la libertad de expresión de la Comisión Afri- 
cana de Derechos Humanos y de los Pueblos (Cadhp), la Organización por la Seguridad y Cooperación en Europa (OSCE) y la OEA, se especifica entre otras cosas que los Estados deben reconocer a los medios comunitarios, establecer procedimientos sencillos para que estos accedan a frecuencias y permitirles financiarse mediante publicidad (OEA, OSCE y CADHP, 2007). La Ley de Comunicación Ecuatoriana cumple en este sentido con varios de aquellos puntos. En primer lugar, si bien como se mencionó antes se reconocía la existencia de medios comunitarios, estos pertenecían al sector público; la ley actual aclara que existen tres sectores, uno de ellos el comunitario (Artículo 70 de la Ley Orgánica de la Comunicación, 2013).

El Artículo 85 de la Ley Orgánica de Comunicación (2013) define de la siguiente manera a los medios comunitarios: "Los medios de comunicación comunitarios son aquellos cuya propiedad, administración y dirección corresponden a colectivos u organizaciones sociales sin fines de lucro, a comunas, comunidades, pueblos y nacionalidades”.

En palabras de José Ignacio López Vigil, representante de la ONG Radialistas Apasionados, ${ }^{3}$ esta ley reconoce la comunicación como un derecho humano universal que supera el ámbito mercantil o estatal.

La distribución tripartita de frecuencias es además uno de los avances más reconocidos. En Ecuador, hasta el 2008, las frecuencias estaban distribuidas en $97 \%$ para privados y $3 \%$ entre comunitarios y públicos (Navas, 2011). Junto con Uruguay; Argentina, con su Ley de Servicios de Comunicación Audiovisual aprobada en 2009, y Bolivia, con su Ley General de Telecomunicaciones, aprobada en 2011, Ecuador se suma a los países con una ley de comunicación que responde a estándares internacionales y genera una distribución de $33 \%$ para medios privados, $33 \%$ para medios públicos y $34 \%$ para medios comunitarios (Artículo 106 de la Ley Orgánica de Comunicación, 2013; Segura, 2014). Esta distribución, según aclara la ley, priorizará al sector comunitario y se alcanzará de manera progresiva.

3 Organización no gubernamental cuya finalidad es promover comunicaciones, especialmente de radio, desde la perspectiva de género y ciudadanía (Radialistas Apasionados, 2014). 
La ley además garantiza que el Estado implementará las políticas públicas necesarias para la creación y el fortalecimiento de medios comunitarios "como un mecanismo para promover la pluralidad, diversidad, interculturalidad y plurinacionalidad” (Artículo 86 de la Ley Orgánica de Comunicación, 2013). Entre estas políticas públicas están el acceso a crédito preferente para la compra de equipos, exenciones de impuestos en importaciones, acceso a capacitación, etc. La implementación de la política será responsabilidad del Consejo de Regulación y Desarrollo de la Información y Comunicación (Cordicom), instancia creada como parte de la Ley Orgánica de Comunicación.

En cuanto a la financiación, la ley garantiza también que los medios puedan financiarse mediante "la venta de servicios y productos comunicacionales, venta de publicidad, donaciones, fondos de cooperación nacional e internacional, patrocinios" (Artículo 87 de la Ley Orgánica de Comunicación, 2013), y además establece que las entidades públicas podrán generar fondos concursables para programas de educación y cultura para este tipo de medios.

\section{Críticas a la Ley de Comunicación en torno a la comunicación comunitaria}

A pesar de los grandes avances en materia de comunicación comunitaria, existen desacuerdos con respecto a la Ley de Comunicación. La sociedad civil, en sus propuestas para una ley democrática, sugirió la creación de un consejo de regulación, en su mayoría formado por representantes de la ciudadanía y la academia, no vinculados al Estado y elegidos por sus méritos, pero con una significativa representación estatal (ALER, 2011). Sin embargo, el Consejo de Regulación y Desarrollo de la Información está conformado según dice actualmente la ley por un representante de la función ejecutiva, quien lo presidirá, un representante de los consejos nacionales de igualdad, un representante del Consejo de Participación Ciudadana y Control Social, un representante de los gobiernos autónomos descentralizados y un representante del defensor del pueblo. Así, queda fuera la academia, que estaba incluida en la propuesta de la sociedad civil, y sobre todo la re- 
presentación ciudadana, que se reduce a un representante del Consejo de Participación Ciudadana y Control Social (López Vigil, 2014).

A pesar de que el Artículo 54 de la LOC expresa la formación de un Consejo Consultivo con un carácter más académico y ciudadano, sus resoluciones están al nivel de la asesoría y no son vinculantes. Es más, hasta la fecha este consejo no se ha conformado.

Dado que la Ley de Comunicación en su Artículo 42 exige el título profesional de periodista para quienes ejercen de manera permanente en los medios de comunicación, José Ignacio López Vigil, director de Radialistas Apasionados, menciona que aquí se evidencia un desconocimiento total de la naturaleza de los medios comunitarios. Un medio comunitario existe por una necesidad de expresión de un grupo, y muchas veces quien trabaja en el medio lo hace de manera voluntaria, obedeciendo su necesidad de comunicar. Por esta razón, la historia de medios comunitarios está llena de ejemplos de comunidades que han logrado con capacitaciones, ayuda de la cooperación internacional y de radios extranjeras, aprender lo necesario para poder elevar su voz sin dejar de lado sus actividades fundamentales del día a día. "A un medio comunitario no se le debería exigir lo mismo que a un medio comercial, en ningún aspecto”, señala López Vigil (2014).

La preocupación de López Vigil tiene una base en los estándares para la libertad de expresión de la Comisión Interamericana de Derechos Humanos, que señalan que "Toda persona tiene derecho a comunicar sus opiniones por cualquier medio y forma. La colegiación obligatoria o la exigencia de títulos para el ejercicio de la actividad periodística, constituyen una restricción ilegítima a la libertad de expresión" (Relatoria Especial para la Libertad de Expresión de la Comisión Interamericana de Derechos Humanos, 2010).

Como se manifestó al principio, los estándares internacionales establecen con claridad que los Estados deberán considerar procedimientos sencillos para la obtención de frecuencias. En Ecuador la adjudicación de frecuencias depende de los concursos a los que el Estado convoca en cada 
provincia. Al final del concurso, de acuerdo con la ley, se remite al Consejo de Regulación y Desarrollo de la Información hasta cinco de los concursantes mejor puntuados.

Para los entrevistados, este mecanismo está planteado al revés y resulta lento. Un medio comunitario que nace como una necesidad debería tener la facilidad de pedir la frecuencia y, si no se encuentran problemas en su proyecto comunicacional y además no existe otro grupo interesado en la frecuencia, la asignación debería ser simple. La Ley de Comunicación ha estado vigente por casi dos años y solo el $4 \%$ de las frecuencias pertenecen ahora al sector comunitario (Cordicom, 2014). Es preciso entender que el proceso es largo por el hecho de que se necesita revertir las frecuencias; sin embargo, los expertos entrevistados sugieren que este proceso podría agilizarse. En Uruguay, Argentina y Colombia la asignación se realiza también mediante concurso abierto con audiencia pública previa. En Argentina, sin embargo, este concurso es permanente, lo que significa que si existen solicitudes inmediatamente se abre un concurso y se tramita la concesión de la frecuencia (Amarc, 2010).

Otro de los problemas que reconocen los entrevistados es que la ley establece que será el Cordicom el encargado de crear políticas públicas para capacitaciones y exención de impuestos, entre otros apoyos, con el fin de que los emprendimientos comunitarios puedan generarse. "Ahí el Cordicom tiene una deuda con los medios comunitarios porque ha pasado ya casi un año y no encuentro ninguna política pública orientada hacia esta acción afirmativa", manifiesta Romel Jurado (2014), abogado y uno de los principales gestores en la redacción de la Ley de Comunicación.

Esto de alguna manera ralentiza también el proceso de redistribución de las frecuencias, pues existen comunidades y organizaciones sociales que quieren acceder a una frecuencia pero no tienen la capacidad de hacerlo. Sin embargo, Cordicom (2014) ha manifestado que está trabajando con la Corporación Nacional de Finanzas Populares y Solidarias y el Servicio de Rentas Internas para sacar adelante estas medidas. 


\section{El Decreto Ejecutivo n. ${ }^{0}$ 16: regulaciones y limitaciones a la sociedad civil}

Ya en 2002 en Ecuador se expidió un reglamento para la aprobación, control y extinción de personas jurídicas de derecho privado, con finalidad social y sin fines de lucro. Posteriormente, en 2008, el Decreto Ejecutivo 982 dispuso que la Secretaría de los Pueblos, Movimientos Sociales y Participación Ciudadana organice, mantenga y difunda el Registro Único de Organizaciones de la Sociedad Civil, ya que antes de esa fecha esta información se encontraba dispersa en diferentes ministerios (Registro Oficial, 2013).

Fue en 2011 cuando el gobierno ecuatoriano consideró necesaria la institucionalización del Sistema Unificado de Información de Organizaciones Sociales; sin embargo, una reforma al reglamento expedido en el 2002 no fue suficiente. Tanto en la ONU y como en la CIDH, ya en 2012 se recomendó velar porque estos reglamentos no bloquearan las labores de las organizaciones en el país, y además se recomendó que se revisaran estas normas, ya que han tenido observaciones por parte de organismos internacionales encargados de temas de derechos humanos (Programa Andino de Derechos Humanos, 2012).

Como consecuencia, en junio de 2013, en el Registro Oficial n. ${ }^{\circ} 19$ se expidió el reglamento para el funcionamiento del Sistema Unificado de Información de las Organizaciones Sociales y Ciudadanas, a través del Decreto Ejecutivo n. ${ }^{\circ} 16$ (Registro Oficial, 2013).

El hecho de que Ecuador fuera el primer país en Latinoamérica en tener una constitución que reconociera el derecho a la comunicación y que además estableciera una redistribución equitativa entre los tres sectores de la comunicación, privado, público y comunitario, es el resultado de la lucha de la sociedad civil. Ecuador está entre los países de la región que agrupan más organizaciones de la sociedad civil dedicadas a la comunicación. Eso, sumado a la movilización de organizaciones indígenas, populares y sociales, consiguió este gran avance registrado en la LOC (Segura, 2014). Muchas de estas organizaciones refutan hoy el decreto que las regula; es decir, el Decreto Ejecutivo n. ${ }^{\circ}$ 16: "El problema no es la regulación, el problema 
son los contenidos" es el criterio de uno de los voceros de la Corporación Participación Ciudadana, una ONG que promueve el involucramiento y la veeduría ciudadana (Jaramillo, 2014).

El fin mismo de una organización de la sociedad civil es la incidencia en las políticas públicas. El ejemplo más claro es la incidencia de las organizaciones dedicadas a la comunicación en la constitución ecuatoriana, pues lograron que se incluyera en ella el derecho al a comunicación. El Artículo 26 del Reglamento para el Funcionamiento del Sistema Unificado de Información de las Organizaciones Sociales y Ciudadanas (conocido como Decreto Ejecutivo n. ${ }^{\circ}$ 16) habla sobre los causales de disolución y establece que dedicarse a actividades de injerencia en políticas públicas que afecten la seguridad del Estado o la paz pública será una de ellas (Registro Oficial, 2013). La interrogante es: ¿cuándo esta incidencia afecta la seguridad del Estado? Para José Ignacio López, es absurdo que esto sea causal de disolución, pues no está claro qué afecta la seguridad del Estado, además de que no existen procesos para apelar.

En el Artículo 7 de las obligaciones de las organizaciones, en el numeral 10, se indica que si una organización es la única en la región debe obligatoriamente aceptar a cualquier persona que tenga interés legítimo de participar en ella. "Eso atenta contra la libre asociación, pues una organización está compuesta de personas que comparten fines y objetivos y por esa razón se unen y no deben tener obligación de aceptar a otras personas dentro de la organización”, manifiesta uno de los voceros de Fundamedios, importante ONG relacionada con el ejercicio de la comunicación en Ecuador (Alarcón, 2014).

Existe una falta de claridad en muchos de los procedimientos que se establecen en el Decreto n. ${ }^{\circ}$ 16. Por ejemplo, el Artículo 28, que habla de la disolución controvertida, expresa que ante oficio o por denuncia en la que se evidencie que la organización ha incurrido en una causal de disolución, esta será cerrada y liquidada (Registro Oficial, 2013). Sin embargo, no se establecen procedimientos a los que la organización pueda recurrir para evitar su cierre. Los estándares internacionales indican que una orga- 
nización social puede ser sancionada, e incluso cerrada, pero señalan que debe haber procesos previos en donde haya derecho a la defensa o en los que exista la posibilidad de rectificar el error. El decreto actual no establece estos procesos, en los que se permita el derecho a la defensa o rectificación (Jaramillo, 2014).

Existen otros problemas que preocupan a los entrevistados, como el control riguroso de actividades y fondos, el proceso de reasignación a nuevos ministerios, las obligaciones que tienen que ver con los espacio de funcionamiento y el patrimonio, la obligación de entregar toda la información solicitada al Estado y otros que, en su opinión, limitan a las organizaciones y ralentizan los procesos de registro. "Los Estados tienen facultad para reglamentar la inscripción, vigilancia y control de organizaciones dentro de sus jurisdicciones, (pero) deben asegurar que los requisitos legales no impidan, retrasen o limiten la creación o funcionamiento de estas organizaciones" (CIDH, 2009, citado por The International Center for Not-forProfit Law, 2014).

Todos estos problemas que consideran los entrevistados afectarían directamente a los nuevos medios comunitarios, pues para que un medio u organización pueda acceder al concurso de frecuencias debe estar conformado jurídicamente como organización social y por tanto regirse según el Decreto n. ${ }^{\circ}$ 16. A su vez, dada la estrecha vinculación entre las organizaciones de la sociedad civil con fondos nacionales o internacionales y la comunicación comunitaria, el control que ahora opera sobre estas organizaciones puede ser susceptible de una intervención estatal, aunque de manera indirecta, sobre el medio comunitario.

\section{Oportunidades y amenazas con el nuevo marco regulatorio}

Como se ha establecido, tanto la Ley Orgánica de Comunicación como el Reglamento para el Funcionamiento del Sistema Unificado de Información de las Organizaciones Sociales y Ciudadanas son parte de este nuevo marco regulatorio que se relaciona directa o indirectamente con la comunicación comunitaria en Ecuador. Con miras a identificar oportunidades y amenazas en torno a este nuevo escenario, se han estudiado dos casos de 
radios consideradas como comunitarias que deberán adecuar su gestión a esta nueva regulación en materia de comunicación.

\section{Caso Radio Sonoonda}

Radio Sonoonda es la radio de la Unión de Asociaciones campesinas del Azuay (Unasay). Se ha mantenido 25 años al aire. La radio está manejada en su mayoría por los miembros de las organizaciones campesinas que forman parte de Unasay. Ellos se encargan desde la dirección hasta la creación de programas.

Radio Sonoonda se constituyó como un medio de comunicación privado debido a que no existía una figura jurídica que amparase a las radios comunitarias. Como expresa Gumucio (2005), el entorno legal y social en los países de Latinoamérica no ha sido nunca el propicio para que se den experiencias de medios comunitarios y participativos; sin embargo, esto no ha impedido crear iniciativas comunitarias como Radio Sonoonda. Si bien la radio se ampara bajo la figura del medio privado, sus objetivos y su esencia se enmarcan dentro de lo comunitario, ${ }^{4} \mathrm{y}$ mantiene en su quehacer diario las directrices y lineamientos de las organizaciones sociales que promovieron su creación.

En la actualidad, Radio Sonoonda se ve — posiblemente — ante su mayor reto en el ámbito institucional: conseguir el reconocimiento como emisora comunitaria, de acuerdo con el nuevo marco que le provee la Ley Orgánica de Comunicación de Ecuador.

Los directivos de Radio Sonoonda han hecho ya la solicitud como organización social Unasay-Fenocin para conseguir la frecuencia como emisora comunitaria. Esta se presenta como una oportunidad sin precedentes para la radio, y sus directivos reconocen que si consiguen la frecuencia podrán acceder a varios beneficios de la ley, por lo cual están efectuando todos los trámites que solicita el Estado.

4 Para un análisis más a fondo de lo que implica una radio comunitaria, se recomienda revisar a Gumucio (2005), Moscoso, Avila y Piedra (2014) y Villamayor (2014). 
El problema más grande en relación con el trámite está en la realización y costos del plan técnico y de sostenibilidad. Gissela Dávila, secretaria ejecutiva de Corape, menciona que este plan tiene que ver solamente con la parte económica, y ahí está el problema, pues la sostenibilidad de un medio comunitario, a criterio de los directivos, tiene que ver con el aspecto social y político y no solo con el económico (Dávila, 2014). Sin embargo, los formularios actuales dejan fuera muchas de estas características del medio comunitario.

El desconocimiento del principio comunitario y la reducción de su sustentabilidad a un análisis financiero hace que medios como Radio Sonoonda encuentren una barrera en el nuevo marco regulatorio. Si ellos no logran superar esta dificultad con el apoyo de asesores técnicos y aportes económicos, no podrán cumplir con todos los requisitos para solicitar una frecuencia como medio comunitario.

\section{Caso Radio La Voz de Ingapirca}

Por otro lado, está el caso de Radio La Voz de Ingapirca. Nació en 1980 de la mano de la Misión Luterana, organización religiosa no gubernamental, y fue una de las primeras cadenas en transmitir programas en quichua.

Para conseguir equipos y para empezar a funcionar contó con el apoyo de organizaciones no gubernamentales, sobre todo noruegas, y de radios extranjeras como Radio Netherlands, que ayudaba con funciones como capacitaciones y programación. Desde hace algunos años, como mencionan sus representantes, las colaboraciones de organizaciones no gubernamentales han disminuido, lo que ha llevado a que se redujera su personal de quince a siete personas. El nuevo marco regulatorio para ONG, en lugar de ser un apoyo, resulta ser una barrera para Radio La Voz de Ingapirca, ya que no hay interés por parte de los organismos internacionales de continuar colaborando en un marco con elevadas restricciones legales.

Hoy en día, esta radio va a renovar su frecuencia como emisora privada y no como comunitaria, renunciando con ello a varios beneficios. De acuerdo con las declaraciones de sus representantes, no quieren estar a merced de la política y poner en riesgo su frecuencia. Para ella, el efecto del nuevo 
marco regulatorio ha levantado sospechas sobre la posibilidad de mantener la independencia editorial, a lo que no está dispuesta a renunciar como organización.

\section{Organizaciones sociales: caso Ecuarunari}

La confederación de pueblos de la nacionalidad kicwhua de Ecuador Ecuarunari nació en 1972 como una organización indígena de la Confederación de Nacionalidades Indígenas del Ecuador (Conaie). Su principal objetivo es propiciar dentro de la población indígena la toma de conciencia para alcanzar la recuperación social, económica y política (Ecuarunari, 2014).

Esta organización social contaba con la colaboración de ONG internacionales importantes como Solidaridad América Latina y Swissaid. Sus integrantes mencionan que hoy en día la colaboración de ONG es reducida y la confederación se mantiene con pocos fondos. Según sus integrantes, esto se ha dado desde la aplicación de los ya nombrados decretos que regulan a la sociedad civil, que poco a poco han dificultado el trabajo de las organizaciones en el país. Además, sus miembros comentan que muchas organizaciones temen trabajar con Ecuarunari por sus posturas de resistencia y oposición.

En 2013, Ecuarunari presentó junto con otras organizaciones de la sociedad civil una demanda de inconstitucionalidad al Decreto n. ${ }^{\circ} 16$, entre otras razones, por atentar contra el derecho de libre asociación. La demanda fue archivada y no ha tenido resolución hasta el presente año.

Como una de las principales organizaciones sociales indígenas de Ecuador, en enero de 2014 Ecuarunari entregó una solicitud para acceder a una frecuencia de emisora comunitaria del espectro radioeléctrico. Sin embargo, el Consejo de Regulación y Desarrollo de la Información no ha convocado aún a un concurso en la región, por lo cual su solicitud no fue atendida. Además, si se convocara ahora al concurso, la confederación no cuenta con los estudios completos debido a dificultades económicas y falta de asesoría. Esta situación dejaría sin acceso a una frecuencia a una organización que apoyó desde el 2000 las propuestas de Corape para conseguir avances en materia de medios comunitarios en la legislación ecuatoriana (ALER, 2011). 
Esto confirma la preocupación de algunos de los expertos consultados, ya que a pesar de que existen emprendimientos comunitarios con la voluntad de surgir, la falta de políticas públicas que apoyen la acción afirmativa encontrada en la ley hace difícil que esto ocurra. No solo Ecuarunari, sino también Radio Sonoonda y Radio Ingapirca, son un ejemplo de esta problemática.

La Ley de Comunicación y el reglamento del Consejo de Regulación y Desarrollo de la Información indican que los medios de comunicación comunitarios son aquellos cuya propiedad, administración y dirección corresponden a colectivos u organizaciones sociales sin fines de lucro, a comunas, comunidades, pueblos y nacionalidades (Ley Orgánica de Comunicación, 2013), es decir, que necesitan personería jurídica para acceder a una frecuencia. Sin la personería jurídica y sin los estudios completos, Ecuarunari no podrá acceder a una frecuencia para emisora comunitaria.

Ecuarunari, junto con otras organizaciones sociales, tiene la intención de realizar un ceremonial público en el cual reconocerán la personería jurídica a organizaciones y comunidades indígenas sin atenerse al decreto, sino aplicando lo que determina la Constitución, el Estado plurinacional y la autodeterminación de los pueblos, para que no solamente deban esperar a que los ministerios les concedan la personería jurídica, sino que las comunidades y confederaciones reconozcan a sus comunidades de base. Todo esto por causa de los problemas que se les ha presentado con el decreto, pues no se les ha reconocido estatutos, los trámites para registrarse han resultado complicados y lentos $y$, como otras organizaciones, tienen temor por las aplicaciones subjetivas que se han dado a algunos de los artículos del Decreto n. ${ }^{\circ} 16$.

\section{Conclusiones y recomendaciones}

Se inició este texto indicando que legislar los medios comunitarios es una tarea difícil que supone un entendimiento profundo de lo que significa la comunicación en su ámbito más amplio, como derecho fundamental. Alfonso Gumucio (2010) afirma que las radios y medios comunitarios han demostrado que responden a una necesidad vital de expresión y que durante 
años funcionaron de manera espontánea. A pesar de las legislaciones duras y la falta de reconocimiento, estas radios se han multiplicado. Su legitimidad proviene de su propio valor como portavoces de la sociedad civil.

El escenario de hoy en día en Ecuador, y de manera paralela en Argentina, Uruguay y Bolivia, muestra una victoria importante: los medios comunitarios están reconocidos legalmente. Sin embargo, ese reconocimiento no puede estar deslindado de su historia y sus características particulares. Es necesario profundizar en este aspecto para continuar creando un escenario favorable para su funcionamiento.

La Ley de Comunicación aprobada en Ecuador ha dado grandes pasos, ya que además de reconocer legalmente a la comunicación comunitaria, establece una redistribución equitativa del espectro radioeléctrico y garantiza el derecho a la comunicación.

A despecho de estos importantes impulsos a la comunicación comunitaria, y a dos años de ejercicio de la nueva ley, el escenario no está libre de dificultades para los medios comunitarios.

Los resultados discutidos en esta investigación comprueban la hipótesis de trabajo en la que se plantea la existencia de un escenario contradictorio entre la Ley de Comunicación y el Decreto Ejecutivo n. ${ }^{\circ} 16$, lo que influye negativamente sobre todo en el fortalecimiento de los medios comunitarios en Ecuador.

No hay una adecuada representación de los medios comunitarios en los espacios de observación y establecimiento de política pública, como Cordicom. La sociedad civil tiene una mínima posibilidad de intervención, prevista únicamente por el miembro designado por el Consejo de Participación Ciudadana. La impresión de los actores y expertos entrevistados es que el Gobierno ha cooptado estos espacios, lo que es un contrasentido para quienes ejercen la comunicación comunitaria como medio alternativo de expresión de la sociedad civil. Esos medios, en muchas ocasiones, llevan agendas editoriales diferentes a las oficialistas, y la consecuencia indirecta de este escenario es que la voz de la sociedad civil sigue sin ser escuchada. 
Ahora bien, es la primera vez que el país atraviesa un proceso de regulación en materia de comunicación. De hecho, en Ecuador nunca antes se había reconocido la existencia de medios comunitarios; por lo tanto, es normal que existan problemas y necesidades de ajustes. Sin embargo, se debe aprender de esos problemas, tener en cuenta las características de los medios comunitarios y sobre todo escuchar a quienes han estado cerca de ellos desde su inicio y conocen sus necesidades (Dávila, 2014): las organizaciones de la sociedad civil, que son la base de la formación de los medios comunitarios, no deberían estar excluidas de este proceso.

La principal preocupación es que si por un lado hay un impulso a los medios comunitarios, por el otro hay una presión sobre las organizaciones en donde nacen estas iniciativas de comunicación comunitaria. Esto deja la impresión de que el sector político ecuatoriano no ha entendido con claridad cuál es la esencia de la comunicación comunitaria y cuáles son sus necesidades. En ello radica la contradicción planteada como hipótesis de esta investigación.

Los medios comunitarios necesitan apoyo en capacitación técnica y financiera. Para quienes han surgido a partir de la espontaneidad, del ingenio y la necesidad de expresión, que les sea exigido un proyecto de comunicación, títulos profesionales, estudios técnicos y otros componentes para solicitar una frecuencia resulta un importante impedimento, que va en desmedro del verdadero propósito de la comunicación comunitaria. La situación sería distinta si, como afirma Romel Jurado, existiera una política pública clara que dé soporte a esta necesidad de apoyo tanto técnico como económico. Una forma de consolidar ese apoyo es dar impulso a la sociedad civil a través de las organizaciones no gubernamentales.

Es necesario reconocer que en el camino por conseguir el reconocimiento legal y público a la comunicación comunitaria, se establecieron elementos para acompañar este proceso que hoy en día no existen. No se puede ver a los medios comunitarios como un tipo más de medios. Su propia esencia los diferencia. 
El escenario legal para los medios de comunicación comunitario no puede estar aislado de los instrumentos y la normativa que regula a la sociedad civil. Estos medios se constituyen como portavoces de la sociedad, por lo tanto no se puede legislar a las organizaciones de la sociedad civil sin influir sobre la situación de medios comunitarios. Si se ponen trabas al proceso de reconocimiento de organizaciones sociales, al mismo tiempo se está poniendo trabas a la creación de medios de estas organizaciones.

Si las organizaciones de la sociedad civil temen los contenidos del Decreto n. ${ }^{\circ} 16$, tendremos medios comunitarios sometidos a esos temores, y por lo tanto carentes de su característica de resistencia y contrapoder. Es necesario tomar en cuenta las recomendaciones internacionales que se han hecho sobre el Decreto n. ${ }^{\circ} 16$ y empezar a apegarse a las dinámicas de la sociedad civil para formular los instrumentos de regulación.

Ecuador ha alcanzado la victoria más grande en cuanto a democratización de la comunicación; los reglamentos, decretos, resoluciones, políticas públicas y demás medidas legales que se elaboren deben responder a este avance y no contradecirlo o limitarlo. Además, considerando que algunos concursos para la obtención de frecuencias están en marcha, Cordicom debe apresurar la formulación de políticas públicas que acompañen el proceso y provean a las organizaciones y medios comunitarios de herramientas para adquirir las frecuencias.

Se debe concretar la creación del Consejo Consultivo que establece la Ley de Comunicación, pues resulta imprescindible la asesoría de la ciudadanía y la academia para acompañar el proceso de creación de medios comunitarios, ya que son quienes conocen más de cerca este tipo de medios.

Con la investigación se ha descrito la situación actual para medios comunitarios en Ecuador bajo los nuevos marcos regulatorios. Si bien las oportunidades han incrementado, existen discordancias que afectan la creación o crecimiento de estos medios, como se estableció en los casos expuestos. Algunos de los problemas no solo radican en la falta de elementos en la Ley de Comunicación o en su falta de aplicación, sino en la influen- 
cia del Decreto n..$^{\circ} 16$ en las organizaciones sociales y por tanto en los medios comunitarios.

La comunicación comunitaria, no solo en Ecuador, sino en Latinoamérica, se ha constituido en un componente clave del sistema medial. Por lo tanto, si se desea continuar con el impulso a los espacios de expresión alternativos y a las voces divergentes cuyas agendas no necesariamente obedecen las posturas oficiales, es fundamental reconocer la necesidad de analizar el entorno desde una perspectiva sistémica (Ávila, 2013). Considerando que la comunicación comunitaria tiene connotaciones que la diferencian de la comunicación tradicional o comercial, la regulación debe incorporar esas importantes diferencias. Esto no solo implica la regulación especializada en comunicación, sino también aquella relacionada con la sociedad civil.

Este análisis del marco regulatorio puede, a partir de la experiencia ecuatoriana, servir como insumo para análisis comparados con otros contextos como el colombiano, el brasileño, argentino, uruguayo y boliviano, cuyas realidades son interesantes por sus experiencias en comunicación comunitaria. Este momento es clave en la medida en que en Latinoamérica se está instalando una discusión sobre los desafíos para la comunicación como parte de un nuevo paradigma que propone Ecuador en materia de regulación y política pública con miras a una verdadera democratización de la comunicación.

\section{Referencias}

Alarcón, M. (14 de septiembre de 2014). Entrevista personal, director de Fundamedios (N. García, entrevistador). Quito.

ALER (2011). Sistematización, Ley de Comunicación en Ecuador. Quito: ALER.

Amarc (2010). Informe anual, diversidad y pluralismo en la radiodifusión. Coyoacán. 
Anderson, P. (1987). Democracia y dictadura en América Latina en la época del setenta. Conferencia de Política Latinoamerica dictada en la Carrera de Sociología.

Araújo, B. (2007). Las voces radiofónicas. Las radios comunitarias en Brasil. Recuperado el 26 de mayo de 2015 de http://hdl.handle. net/10803/10318

Ávila, C. (2013). El sistema de comunicación en la Ley de Comunicación en Ecuador. Chasqui, 124, 71-79.

Baxter, P. y Jack, S. (2008). Qualitative case study methodology: Study design and Implementation for novice researchers. The Qualitative Report, 13(4), 544-559.

Celis, C. y Sánchez, P. (2011). Desplazamientos discursivos. Delo estudiantil a lo juvenil. Neoliberalismo y ONG en América Latina, Caso Ecuador. Revista del Centro Telúrico de Investigaciones Teóricas, 1.

CIDH (2009). Informe sobre democracia y derechos humanos en Venezuela. Recuperado el 15 de septiembre de 2014 de http: / / www.cidh.oas. org/ countryrep/Venezuela2009sp/VE09CAPVSP.htm

Cordicom (2014). Ecuador es ejemplo en democratización de la comunicación a través de medios comunitarios. Recuperado el 15 de septiembre de 2014 de www.cordicom.gob.ec: http://www.cordicom.gob.ec/john-dinges-ecuador-es-ejemplo-en-democratizacion-de-la-comunicacion-a-traves-de-medios-comunitarios/

Corrales, F. y Hernández, H. (2009). Comunicación alternativa en nuestros días. Razón y Palabra, 70.

Dagnino, E. (2011). Civil society in Latin America: Participation citizens or service providers. En Press, O. U. y Edwards, M. (ed.), Oxford Handbook of Civil Society. Oxford. 
Dávila, G. (2014). Punto Noticias, Pichincha Universal.

Ecuarunari (2014). Ecuarunari, Confederación Kichwa del Ecuador. Recuperado el 22 de Septiembre de 2014 de http://ecuarunari.org/ portal/info/historia

Flyvbjerg, B. (2006). Five misunderstandings about case-study research. Qualitative Inquiry, 12(2), 219-245.

Gueerts, A., Van Oeyen, V. y Villamayor, C. (eds.) (2004). La práctica inspira. Quito: ALER-Amarc.

Gumucio, A. (2005). Arte de equilibristas: la sostenibilidad de los medios de comunicación comunitarios. Punto Cero, 10(10), 6-19.

Gumucio, A. (2010). Identidad, políticas y legislación de la radio local y comunitaria. En Herrera, K. y Gumucio, A., Políticas y Legislación para la radio local en América Latina (p. 225). Bolivia: Plural Editores.

Jaramillo, S. (6 de septiembre de 2014).Entrevista personal, subdirector de Corporación Participación Ciudadana (N. García, entrevistador). Quito.

Jelin, E. (1994). Ciudadanía emergente o exclusión. Movimientos sociales y ONG en los 80. Revista Mexicana de Sociología, 56, 91-108.

Jurado, R. (14 de septiembre de 2014). Entrevista personal, asesor legislativo (N. García, entrevistador). Quito.

King, G., Keohane, R.y Verba, S. (1994). Designing social inquiry. Scientific inference in qualitative research. Princeton: Princeton University Press.

Ley Orgánica de Comunicación (25 de Junio de 2013).Tercer Suplemento del Registro Oficial No. 022. Quito. 
López Vigil, J. I. (5 de agosto de 2014). Entrevista personal, director de Radialistas Apasionados (N. García, entrevistador). Quito.

Milan, S. (2006). Medios comunitarios y regulación. Una perspectiva de comunicación para el desarrollo. Investigación y Desarrollo, 14(2), 268-291.

Moscoso, V., Ávila, C. y Piedra, J. (2014). Comunicación comunitaria y radios universitarias: Un punto de convergencia. Convergencia de pantallas, diversidad de visiones. Loja: Universidad Técnica Particular de Loja.

Navarro, D. (2011). Transformando el espacio público. El trabajo de una radio local religiosa en una comunidad urbana marginal. En Krohling, C., Tufte, T. y Vega, J. (eds.), Trazos de una otra comunicación en América Latina (pp. 92-108). Barranquilla: Universidad del Norte.

Navas, M. (2011). Espectro abierto para el desarrollo. Estudio de caso: Ecuador. Ciespal.

OEA, OSCE y CADHP (2007). Declaración conjunta sobre diversidad en la radiodifusión. Relatoría especial para libertad de expresión. Recuperado en septiembre de 2014 de http://www.oas.org/es/cidh/ expresion/showarticle.asp? $\operatorname{artID}=719 \&$

Programa Andino de Derechos Humanos (2012). Informe de Derechos Humanos. Quito: Universidad Andina Simón Bolívar.

Radialistas Apasionados (2014). Sobre Radialistas / Quiénes somos. Recuperado el 2 de noviembre de 2014 de http: / /www.radialistas.net/ sobre-radialistas/quienes-somos

Registro Oficial (2013). Reglamento para el funcionamiento del sistema unificado de información de las organizaciones sociales y ciudadanas, 019. Quito. 
Relatoría Especial para la Libertad de Expresión de la Comisión Interamericana de Derechos Humanos (2010). Estándares de Libertad de expresión para una radiodifusión libre e incluyente. Recuperado el 14 de septiembre de 2014 de http://www.oas.org/es/cidh/expresion/docs/cd/sistema_interamericano_de_derechos_humanos/index_ELERLI.html

Rodríguez, C. (2009). De medios alternativos a medios ciudadanos. Folios, 21 y 22, 13-25.

Sáez, C. (2008). Tercer sector de la comunicación. Teoría y praxis de la televisión alternativa. Bellatera: Universidad Autónoma de Barcelona.

Segura, M. S. (2012). La sociedad civil ecuatoriana en la disputa por una nueva ley de comunicación. Comparación con el proceso argentino. I Coloquio de Comunicación para la Transformación Social. Córdova.

Segura, M. S. (2014). La Sociedad Civil y la democratización de las comunicaciones en Latinoamérica. Íconos, Revista de ciencias sociales, 49, 65-80.

The International Center for Not-for-Profit Law (ICNL) (2014). El decreto 16 en el contexto del Derecho Internacional. En Montufar, C., Herrería, E., Pérez, C.Tenesaca, D. y Fundamedios (eds.), El derecho a reunirnos en paz. El Decreto 16 y las amenazas a la organización social en el Ecuador. Quito: Canvas Publicidad.

Villamayor, C. (2014). Radios comunitarias, gestoras de procesos comunicacionales. Mediaciones, 12, 88-103.

Yin, R. K. (2009). Case study research design and methods. Thousand Oaks: Sage Publications. 